\title{
Mortalité par cause en fonction du niveau de compétence professionnelle au Canada : une étude de suivi sur 16 ans
}

\author{
M. Tjepkema, M.H.P. (1); R. Wilkins, M. Urb. (1, 2); A. Long, M.A. (3)
}

Cet article a fait l'objet d'une évaluation par les pairs.

\section{Résumé}

Introduction : Les données sur la mortalité par profession n'étant pas facilement accessibles au Canada, nous avons analysé des données issues du recensement pour étudier les taux de mortalité par cause au sein de différents groupes de professions hiérarchisés par niveaux de compétence.

Méthodologie : Un échantillon de 15 \% des répondants de 25 ans et plus au recensement du Canada de 1991 avait été précédemment couplé avec 16 années de données sur la mortalité (1991-2006). Notre analyse est fondée sur une cohorte de 2,3 millions de personnes âgées de 25 à 64 ans au début de l'étude, au sein de laquelle 164332 décès ont été enregistrés au cours de la période de suivi. Les professions ont été classées conformément à la Classification nationale des professions et ont été réparties en cinq groupes de niveaux de compétence. Les taux de mortalité normalisés selon l'âge (TMNA), les rapports de taux (RT), les différences de taux (DT) et la surmortalité ont été calculés par niveau de compétence professionnelle pour différentes causes de décès.

Résultats : Les TMNA variaient clairement selon le niveau de compétence : ils étaient plus élevés chez les personnes occupant un poste non spécialisé (et chez celles sans emploi) et moins élevés chez celles occupant un poste professionnel. Chez les hommes, les RT toutes causes confondues étaient de 1,16,1,40,1,63 et 1,83 à mesure que le niveau de compétence professionnelle diminuait et en référence au groupe des professionnels. Chez les femmes, le gradient était moins prononcé : 1,23, 1,24, 1,32 et 1,53. Nous avons observé ce gradient pour la plupart des causes de décès. Les RT concernant les niveaux de compétence les plus faibles par rapport aux plus élevés étaient supérieurs à 2 pour le VIH/sida, le diabète sucré, le suicide et le cancer du col de l'utérus, ainsi que pour les causes de décès associées au tabagisme et à la consommation excessive d'alcool.

Conclusion : Les gradients de la mortalité par niveau de compétence professionnelle étaient clairs pour la plupart des causes de décès. Ces résultats fournissent des indicateurs de référence détaillés sur la mortalité par cause qui n’étaient pas disponibles au Canada auparavant.

Mots-clés : statut socioéconomique, mortalité différentielle, niveau de compétence professionnelle, Canada

\section{Introduction}

La relation entre la profession d'un individu et la mortalité est bien connue. Les résultats de l'étude Whitehall ont révélé un gradient social inverse : le nombre de décès par coronopathie était plus élevé chez les fonctionnaires britanniques qui occupaient un poste nécessitant peu ou pas de compétences, et moins élevé chez les fonctionnaires qui occupaient un poste nécessitant davantage de compétences, un niveau de scolarité plus élevés ou d'autres qualifications $^{1}$. Des gradients sociaux similaires relatifs à la mortalité ont été observés dans d'autres pays et pour d'autres professions ${ }^{2-7}$.

Le lien entre la santé et la profession est complexe. On a émis l'hypothèse que les professions avaient une incidence sur la santé en raison de facteurs matériels et psychosociaux et de l'exposition à des conditions ou des matières dangereuses sur les lieux de travaill-12 ${ }^{7}$ Par exemple, les personnes exerçant les professions les plus spécialisées, lesquelles ont tendance à être mieux rémunérées, peuvent avoir un meilleur accès à des ressources matérielles favorisant les pratiques saines en matière de santé, comme un logement et une nourriture de bonne qualité. En outre, la profession peut avoir une influence positive ou négative sur la santé en fonction des exigences et des gratifications particulières associées à différents types de travail : réseaux sociaux, stress lié au travail, degré d'autonomie et de contrôle par rapport aux conditions de travail ${ }^{9,10,12-14}$. L'exposition à des matières dangereuses sur les lieux de travail varie elle aussi en fonction de la profession et contribue aux écarts entre taux de mortalité.

Au Canada, les études de grande envergure basées sur une population et portant sur la mortalité par profession sont moins fréquentes qu'ailleurs. Cela s'explique en partie par le fait que l'information sur la profession habituellement incluse dans les

Rattachement des auteurs :

1. Division de l'analyse de la santé, Statistique Canada, Ottawa (Ontario), Canada

2. Département d'épidémiologie et de médecine communautaire, Université d'Ottawa, Ottawa (Ontario), Canada

3. Agence de la santé publique du Canada, Ottawa (Ontario), Canada

Correspondance : Michael Tjepkema, Division de l'analyse de la santé, Statistique Canada, RHC-24Q, 100, promenade Tunney's Pasture, Ottawa (Ontario) K1A 0T6; tél. : 613-951-3896; téléc. : 613-951-3959; courriel : michael.tjepkema@statcan.gc.ca 
enregistrements de décès de la plupart des provinces n'est pas saisie ou codée dans un format lisible par machine. Plusieurs études de suivi fondées sur le couplage de dossiers ont néanmoins examiné le lien entre profession et mortalité, chacune faisant ressortir des taux de mortalité plus élevés chez les professions exigeant des niveaux de compétence plus faibles ${ }^{15-18}$. Leurs résultats ont été limités en raison de la proportion de la population étudiée (géographiquement ou par âge, sexe ou profession), la petite taille des échantillons, le manque d'information à propos des causes de décès ou une combinaison de ces facteurs.

Récemment, des données de recensement provenant d'un échantillon de $15 \%$ de la population canadienne âgée de 25 ans et plus ont été associées à des données sur la mortalité sur une période d'environ 16 ans $^{19,20}$. Les résultats des 11 premières années de suivi ont révélé que les taux de mortalité globaux et les taux de mortalité liée au suicide, aux blessures accidentelles et à des causes susceptibles d'être évitées par intervention médicale diminuaient graduellement à mesure que le niveau de compétence professionnelle augmentait ${ }^{19,21-23}$. Cependant, ces résultats n'ont pas été analysés en utilisant un large éventail de causes de décès.

L'objectif de notre analyse est d'utiliser l'ensemble des données interreliées sur une période de 16 ans pour étudier, en fonction des groupes de causes de décès de l'étude Charge mondiale de morbidité, les taux de mortalité par niveau de compétence professionnelle chez les membres de la cohorte âgés de 25 à 64 ans au début de l'étude, ainsi que d'examiner les causes de décès selon trois facteurs de risque (tabagisme, consommation d'alcool et consommation de drogues) et les décès avant l'âge de 75 ans susceptibles d'être évités par intervention médicale.

\section{Méthodologie}

\section{Source de données}

Notre étude repose sur une analyse secondaire des données de 1991 à 2006 issues de l'Étude canadienne de suivi de la mortalité et du cancer selon le recensement ${ }^{19,20}$.
Pour être admissible à la cohorte, il fallait être âgé de 25 ans ou plus et avoir rempli le questionnaire long du recensement de 1991, ce qui excluait les personnes vivant en établissement (p. ex. hôpitaux, maisons de soins infirmiers et prisons). Pour faire l'objet d'un suivi de la mortalité, les répondants au recensement visés par l'étude devaient d'abord être couplés à un fichier de noms crypté extrait des données non financières des déclarants. Environ 80 \% des répondants au recensement visés par l'étude ( $n=2860244$ ) ont été appariés avec le fichier de noms. Un échantillon aléatoire de répondants ( $\mathrm{n}=125$ 409) a ensuite été retiré pour réduire la taille de la cohorte finale ( $\mathrm{n}=2734835$ ) à $15 \%$ de la population canadienne âgée de 25 ans ou plus au recensement de 1991, comme le précise le protocole sur le couplage des enregistrements. Cette cohorte a ensuite été couplée à la Base canadienne de données sur la mortalité (du 4 juin 1991 au 31 décembre 2006) à l'aide de méthodes de couplage probabiliste fondées principalement sur les noms et les dates de naissance ${ }^{24}$. En l'absence de couplage avec les enregistrements de décès, on a généralement pu déterminer le statut relatif au suivi (vivant, décédé, émigré ou perdu de vue) à partir des données sur les déclarants ${ }^{19,20}$. Notre analyse s'est limitée aux personnes âgées de 25 à 64 ans au début de l'étude ( $\mathrm{n}=2312$ 400). Dans cet échantillon, environ 2 millions de personnes exerçaient une profession codée et $6 \%$ d'entre elles sont décédées au cours de la période de suivi. Environ 313400 membres de la cohorte des 25-64 ans n'exerçaient pas de profession codée. Le tableau 1 présente les effectifs de la cohorte, les années-personnes à risque et le nombre de décès en fonction du niveau de compétence professionnelle, du groupe d'âge et du sexe.

\section{Définitions}

Les occupations ont été codées en fonction du type de travail qu'exerçaient les répondants dans la semaine précédant le recensement de 1991 ou, dans le cas où les personnes étaient sans emploi au cours de la semaine en question, en fonction de l'emploi qu'elles avaient occupé le plus longtemps depuis le $1^{\text {er }}$ janvier 1990. On a demandé aux répondants d'indiquer le type de travail qu'ils exécutaient, de même que les activités ou tâches les plus importantes de ce travail ${ }^{25}$. Cette information a ensuite été codée selon les catégories professionnelles de la Classification nationale des professions de $1990^{26}$. Le niveau de compétence de chacune des professions a été ensuite associé à l'une des catégories suivantes : poste professionnel, poste de gestion, poste spécialisé/ technique/de supervision, poste de spécialisation intermédiaire et poste non spécialisé. Le " niveau de compétence » est défini de façon générale comme la quantité et le type de scolarité et de formation exigés pour accéder à un poste et exécuter les tâches qui y sont rattachées. Dans la Classification nationale des professions, aucun niveau de compétence n'a été associé aux postes de gestion, car certains facteurs autres que la scolarité et la formation (par exemple l'expérience préalable) sont souvent plus déterminants à l'embauche à de tels postes. Pour les fins de notre étude, les gestionnaires ont été classés entre les catégories "poste professionnel » et " poste de supervision ». Les répondants qui n'ont pas travaillé au cours de la période de référence ont été classées dans une catégorie à part, " sans profession ", qui comprend les chômeurs de longue date, les étudiants adultes, les parents au foyer, les personnes dans l'incapacité de travailler et toute autre personne n'ayant pas travaillé au cours de la période de référence.

\section{Techniques d'analyse}

Pour chacun des membres de la cohorte, des jours-personnes de suivi ont été calculés pour la période allant du début du recensement (le 4 juin 1991) à la date du décès, celle de l'émigration ou celle de la fin de l'étude (le 31 décembre 2006). Ensuite, les années-personnes à risque ont été calculées en divisant les jours-personnes de suivi par 365,25. Pour calculer les taux de mortalité normalisés selon l'âge (TMNA), on a utilisé les taux de mortalité selon l'âge au début de l'étude, le sexe et le niveau de compétence professionnelle par groupe d'âge de cinq ans, la structure démographique de la cohorte (années-personnes à risque) sans 
TABLEAU 1

Membres de la cohorte, années-personnes à risque et décès déterminés, selon le groupe d'âge, le sexe et le niveau de compétence professionnelle au début de l'étude, Canada, 1991-2006

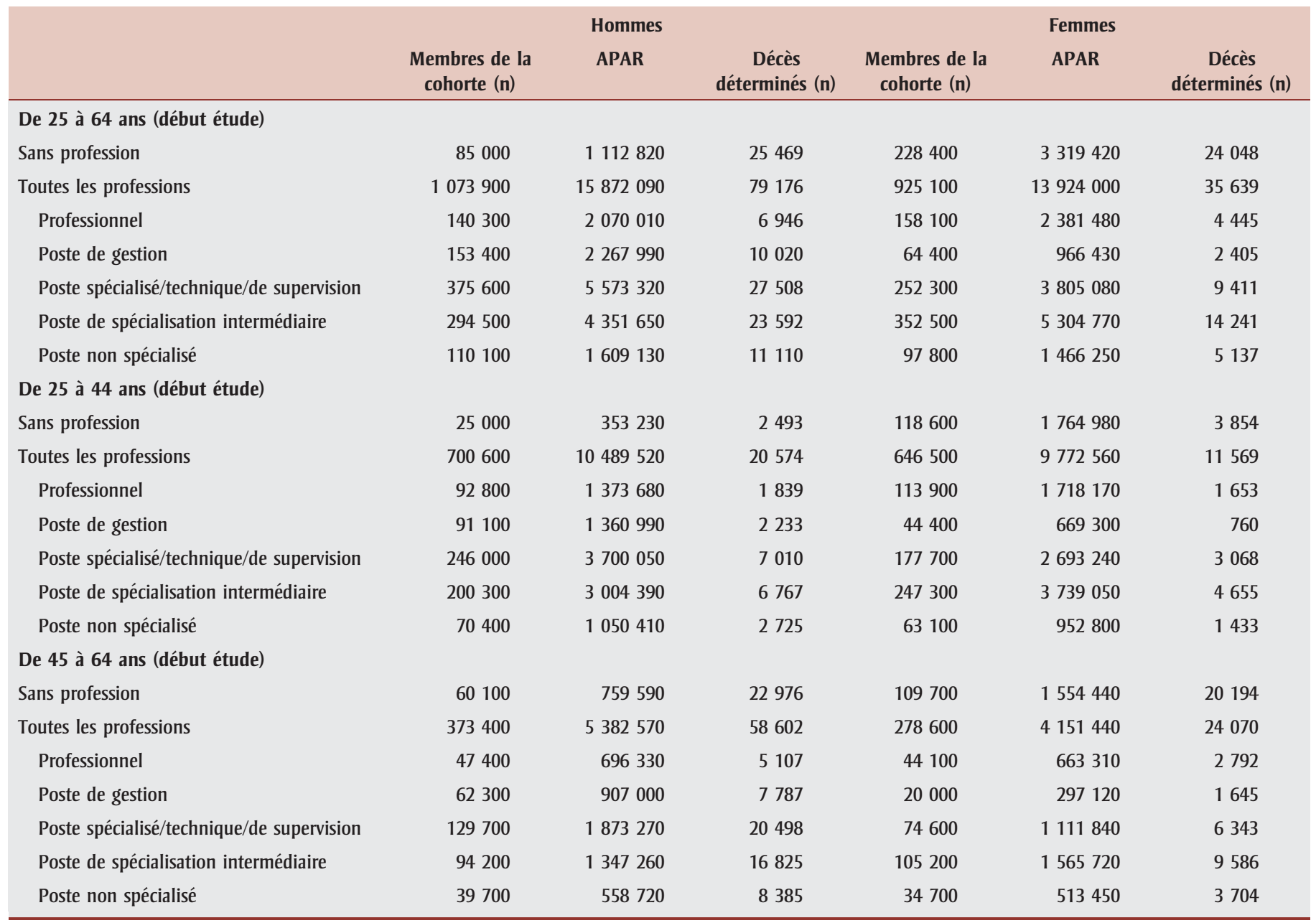

Source : Étude canadienne de suivi de la mortalité et du cancer selon le recensement, 1991-2006 ${ }^{20}$.

Abréviation : APAR, années-personnes à risque.

égard au sexe servant de population de référence.

Les inégalités relatives ont été évaluées en fonction des rapports de taux (RT) et de la surmortalité en pourcentages. Les RT ont été calculés en divisant le TMNA d'un niveau professionel spécifique (poste non spécialisé, poste de spécialisation intermédiaire, poste spécialisé/technique/de supervision, poste de gestion) par le TMNA des personnes occupant un poste professionnel. Un RT supérieur à 1,00 indique un risque de mortalité accru. Pour calculer la surmortalité en pourcentage, on a soustrait le TMNA des personnes occupant un poste professionnel du TMNA calculé pour l'ensemble de la cohorte, sans égard à la profession, puis divisé ce résultat par le TMNA de tous les membres de la cohorte actifs sur le plan professionnel et multiplié le tout par 100 .

Les inégalités absolues ont été évaluées au moyen des différences de taux (DT) et de la surmortalité absolue. Les DT ont été calculées en soustrayant le TMNA des personnes occupant un poste non spécialisé, un poste de spécialisation intermédiaire, un poste spécialisé/technique/de supervision ou poste de gestion, respectivement, du TMNA calculé pour les personnes occupant un poste professionnel. Une DT supérieure à zéro indique une surmortalité. Pour calculer la surmortalité absolue, nous avons soustrait le TMNA des personnes occupant un poste professionnel du TMNA de tous les membres de la cohorte exerçant une profession. La différence représente le nombre de décès (pour 100 000) qui auraient hypothétiquement pu être évités si tous les membres de la cohorte exerçant une profession avaient présenté les taux de mortalité des personnes occupant un poste professionnel.

Les intervalles de confiance (IC) à $95 \%$ des TMNA, des RT et des RD ont été calculés en se fondant sur des méthodes décrites dans un ouvrage paru antérieurement ${ }^{27}$.

Les données sur la mortalité comprenaient la cause sous-jacente de décès, codée selon la CIM-9 (Classification internationale des maladies, $9^{e}$ révision ${ }^{28}$ ) pour les décès avant 2000, et selon la CIM-10 (Classification internationale des maladies, $10^{e}$ révision ${ }^{29}$ ) pour les décès entre 2000 et 2006 . Les décès ont été regroupés selon les catégories de l'étude sur la 
Charge mondiale de morbidité (mise à jour de 2004) ${ }^{30}$. En se fondant sur les définitions usuelles, les causes de décès ont été regroupées par facteurs de risque comportementaux, à savoir les maladies associées au tabagisme ${ }^{2}$ (p. ex. cancer de la cavité buccale, du pharynx, de l'oesophage, du larynx, de la trachée, des bronches et du poumon, maladie pulmonaire obstructive chronique), à la consommation d'alcool ${ }^{2}$ (p. ex. psychose alcoolique, cirrhose alcoolique du foie et pancréatite, empoisonnement accidentel par l'alcool) et à la consommation de drogues ${ }^{31}$ (p. ex. empoisonnement accidentel par les narcotiques et autres drogues, toxicomanie). Nous avons aussi examiné, chez les personnes de moins de 75 ans, les décès susceptibles d'être évités par intervention médicale, comme les décès dus à une maladie vasculaire cérébrale, à une hypertension, à un cancer du sein ou à une pneumonie ou une influenza ${ }^{2,32}$. La définition détaillée des groupes de causes est disponible sur demande.

L'Étude canadienne de suivi de la mortalité et du cancer selon le recensement a été approuvée par le Comité des politiques de Statistique Canada après consultation du
Comité de la confidentialité et des mesures législatives de Statistique Canada, de la Division des services d'accès et de contrôle des données et du Commissaire à la protection de la vie privée du Canada.

\section{Résultats}

Sur les 2,3 millions de membres de la cohorte âgés de 25 à 64 ans au début de l'étude, $7 \%$ des hommes et $20 \%$ des femmes n'exerçaient aucune profession codée par le recensement. Sur les 2 millions de membres de la cohorte ayant déclaré exercer une profession, $13 \%$ des hommes et $17 \%$ des femmes occupaient un poste professionnel; $14 \%$ des hommes et $7 \%$ des femmes occupaient un poste de gestion; $35 \%$ des hommes et $27 \%$ des femmes occupaient un poste spécialisé, technique ou de supervision et $27 \%$ des hommes et $38 \%$ des femmes occupaient un poste de spécialisation intermédiaire. Le reste des hommes (10 \%) et des femmes (11 \%) occupaient un poste non spécialisé (tableau 1).

Comme l'illustre le tableau 2, les TMNA toutes causes de décès confondues, et pour les deux sexes, variaient en fonction du niveau de compétence professionnelle, les taux de mortalité plus élevés ayant été observés chez les personnes exerçant une profession moins spécialisée. Comparativement aux hommes occupant un poste professionnel, les RT étaient de 1,16 pour ceux occupant un poste de gestion, de 1,40 pour ceux occupant un poste spécialisé, technique ou de supervision, de 1,63 pour ceux occupant un poste de spécialisation intermédiaire et de 1,83 pour ceux occupant un poste non spécialisé. Chez les femmes, les RT correspondants étaient respectivement de 1,23, de 1,24, de 1,32 et de 1,53. Chez les personnes sans profession, les RT étaient de 3,57 pour les hommes et de 2,20 pour les femmes. Les DT servant à comparer les personnes occupant un poste professionnel à celles occupant un poste avec d'autres niveaux de compétence professionnelle étaient les plus élevées pour ce qui était des professions non spécialisées (308 pour 100000 chez les hommes; 126 pour 100000 chez les femmes).

Le gradient de mortalité par niveau de compétence professionnelle variait en fonction des groupes de causes de décès (tableaux 3 et 4). Chez les hommes, les RT comparant ceux qui occupaient un poste

TABLEAU 2

Nombre de décès, taux de mortalité normalisés selon l'âge pour 100000 années-personnes à risque, rapports de taux et différences de taux, selon le niveau de compétence professionnelle et le sexe, membres de la cohorte âgés de 25 à 64 ans au début de l'étude, Canada, $1991-2006$

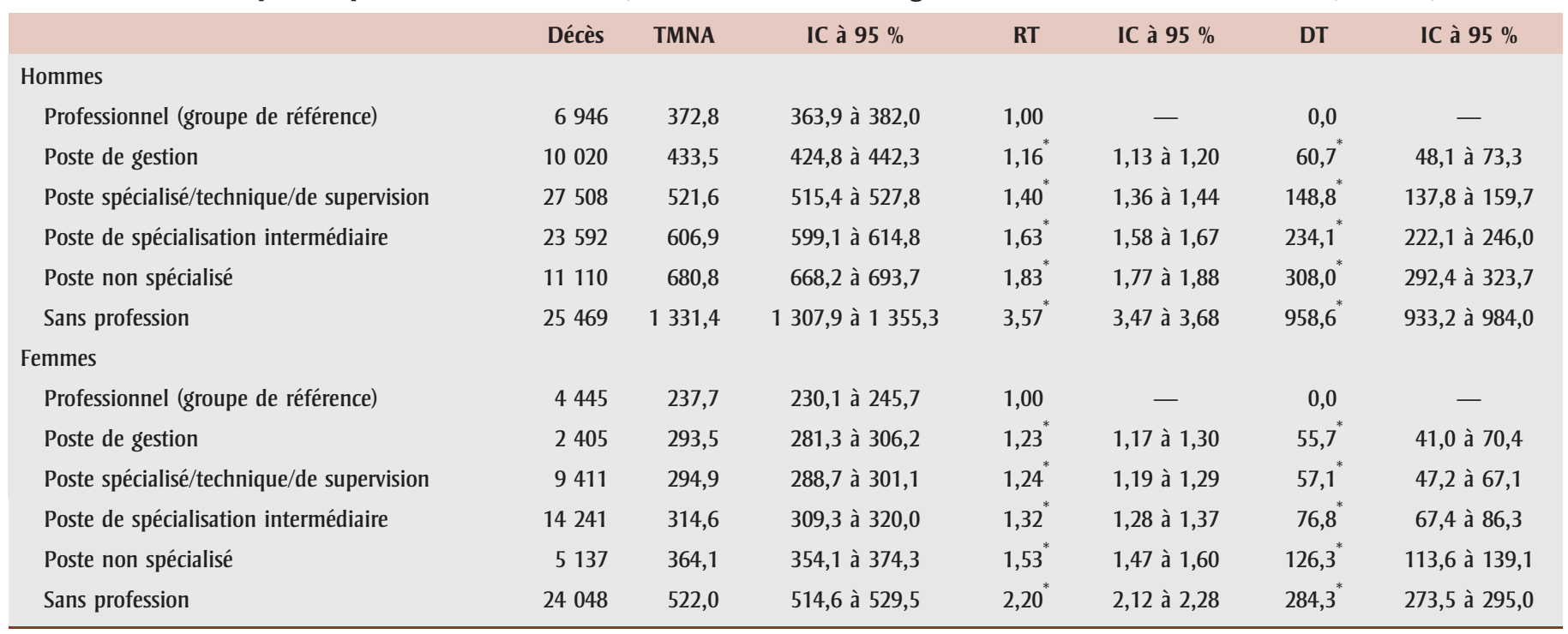

Source : Étude canadienne de suivi de la mortalité et du cancer selon le recensement, 1991-2006 ${ }^{20}$.

Abréviations : DT, différence de taux; IC, intervalle de confiance; RT, rapport de taux; TMNA, taux de mortalité normalisé selon l'âge.

Remarques : La population type (années-personnes à risque) pour la normalisation selon l'âge est extraite de la répartition de la cohorte selon l'âge (tranches d'âge de 5 ans).

— : sans objet.

* Écart significatif par rapport aux professionnels $(p<0,05)$. 


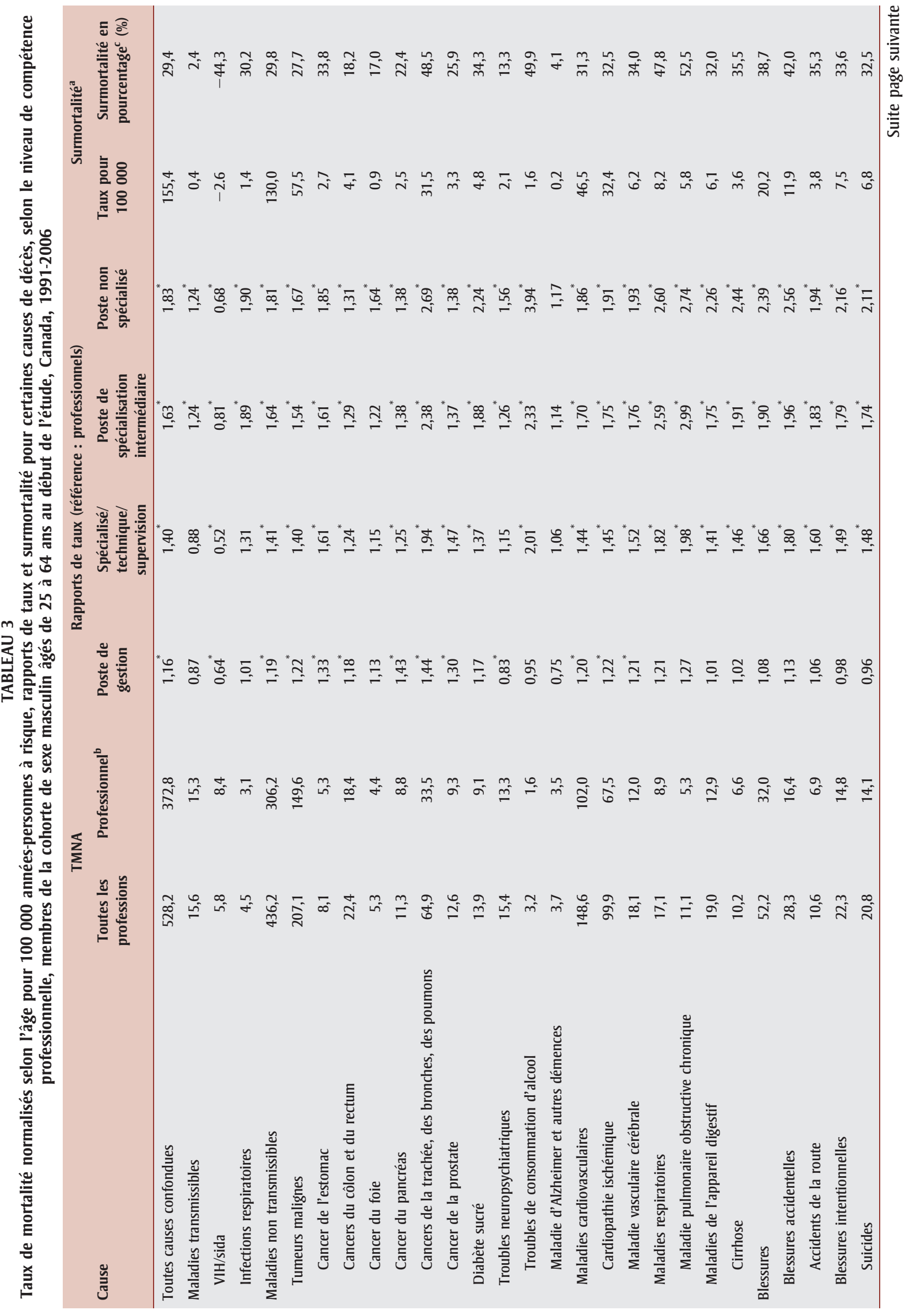




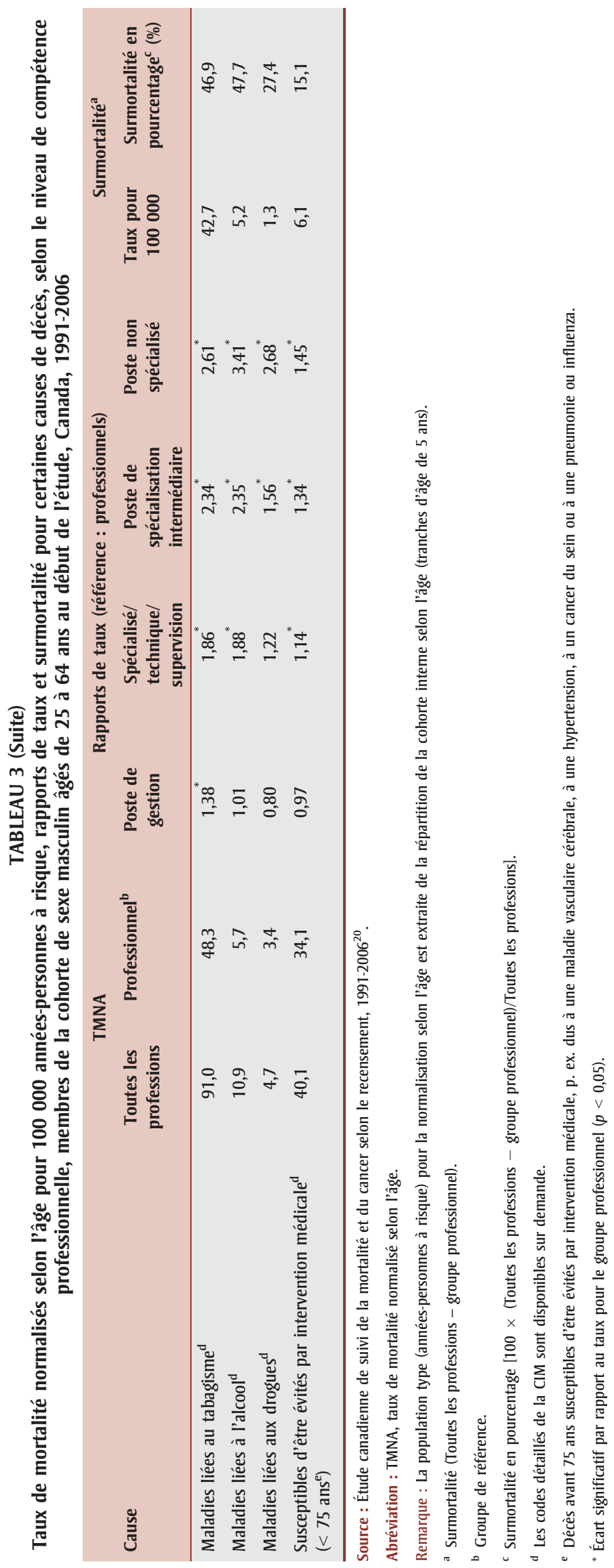

non spécialisé et ceux qui occupaient un poste professionnel était supérieur à 2 pour les décès dus aux troubles liés à la consommation d'alcool $(3,94)$, à une maladie pulmonaire obstructive chronique $(2,74)$, aux cancers de la trachée, des bronches et du poumon $(2,69)$, aux blessures accidentelles $(2,56)$, à une cirrhose $(2,44)$, au diabète sucré $(2,24)$ et au suicide $(2,11)$ (tableau 3). En revanche, le gradient était inversé pour les décès par VIH/sida $(0,68)$ et il n'était pas statistiquement significatif pour la démence $(1,17)$.

Chez les femmes, le RT de comparaison entre celles qui occupaient un poste non spécialisé et celles qui occupaient un poste professionnel était supérieur à 2 pour les décès dus au cancer du col de l'utérus $(3,19)$, au diabète sucré $(2,54)$, aux troubles liés à la consommation d'alcool $(2,42)$, à une cardiopathie ischémique $(2,29)$, aux cancers de la trachée, des bronches et du poumon $(2,24)$, à une maladie pulmonaire obstructive chronique $(2,06)$ et à une cirrhose $(2,05)$ (tableau 4$)$. En revanche, le gradient était inversé pour les décès par cancer du sein $(0,85)$ et il n'était pas statistiquement significatif pour le cancer de l'estomac $(1,35)$, la démence $(1,28)$, les infections respiratoires $(1,24)$, les cancers du côlon et du rectum $(1,13)$ et le cancer des ovaires $(1,35)$.

La proportion de surmortalité associée au niveau de compétence professionnelle figure dans la dernière colonne des tableaux 3 et 4 . Si tous les membres de la cohorte actifs sur le plan professionnel avaient affiché les TMNA de ceux qui occupaient un poste professionnel, le TMNA toutes causes confondues aurait été de $29 \%$ plus faible chez les hommes et de $21 \%$ plus faible chez les femmes, ce qui représente respectivement 155 et 64 décès de moins pour 100000 annéespersonnes à risque. Environ la moitié de cette surmortalité est due à des décès liés aux maladies cardiovasculaires et à des décès liés aux cancers de la trachée, des bronches et du poumon.

Les causes de décès ont également été regroupées par facteur de risque (maladies liées au tabagisme, maladies liées à la consommation d'alcool et maladies liées à 


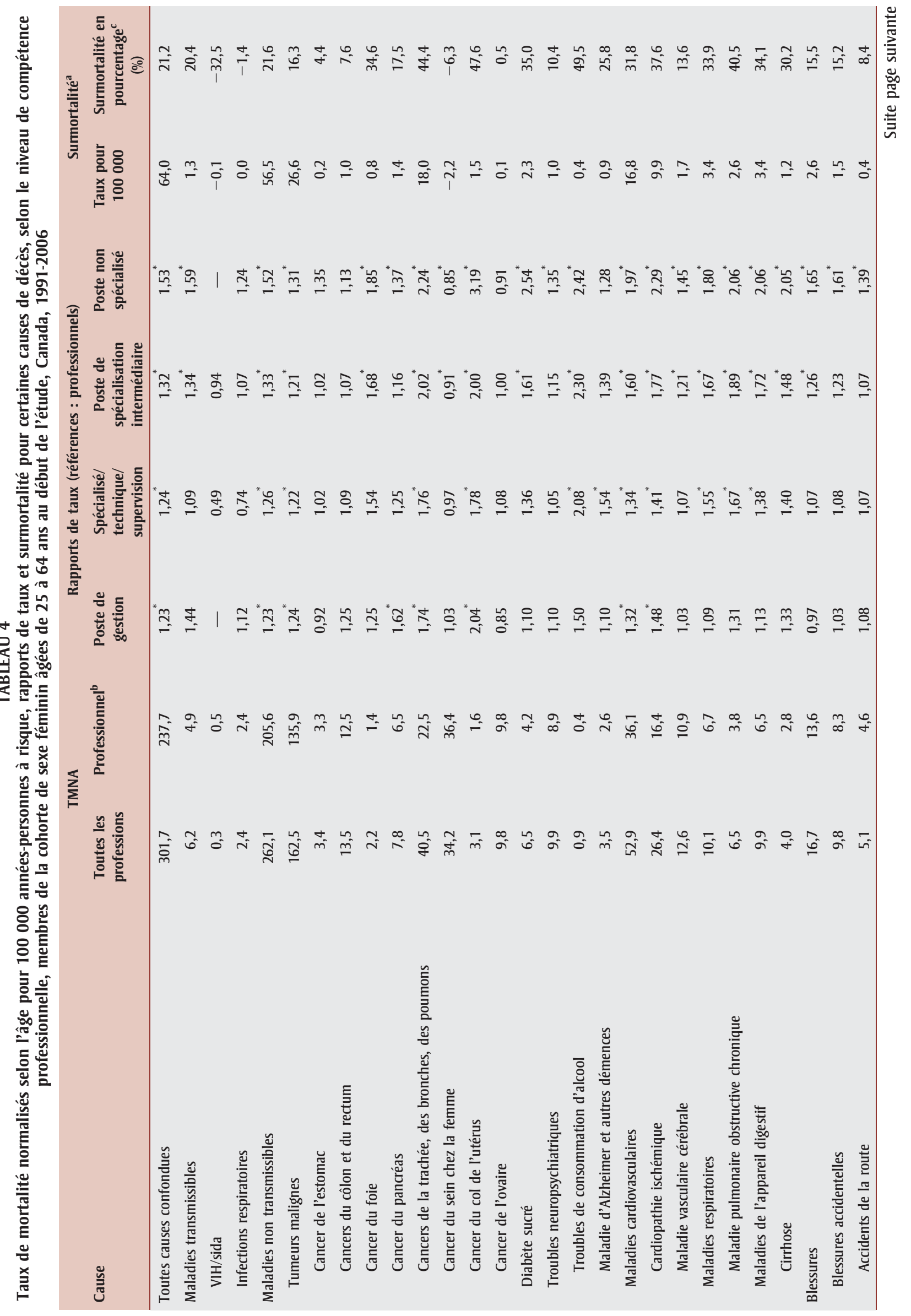




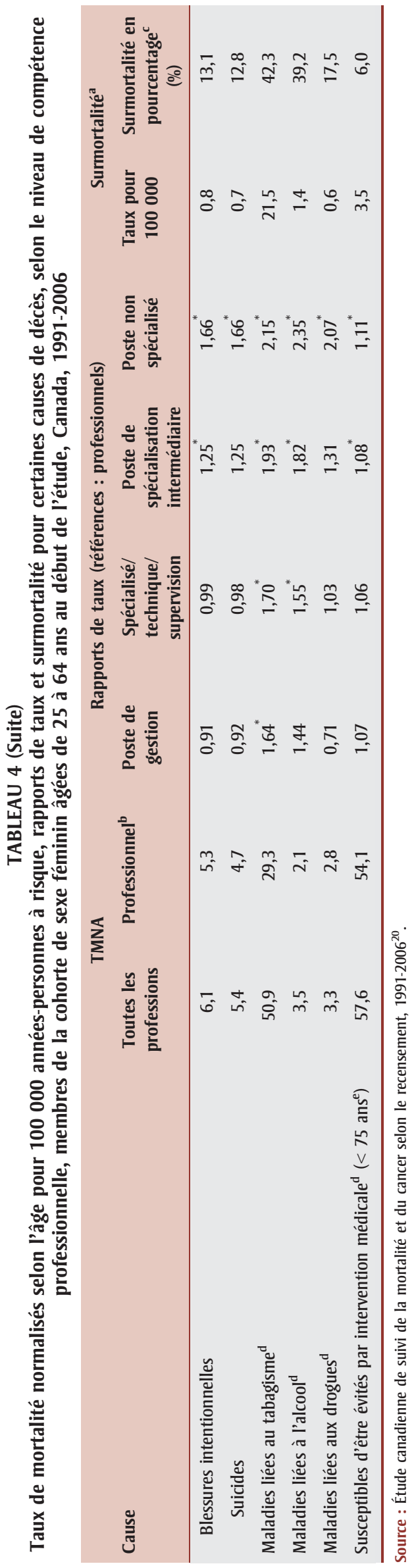

la consommation de drogues). Pour les maladies liées au tabagisme, le RT était de 2,61 pour les hommes qui occupaient un poste non spécialisé comparativement à ceux qui occupaient un poste professionnel (tableau 3). Chez les femmes, le RT correspondant était de 2,15 (tableau 4). Les RT pour les décès dus à une maladie liée à la consommation d'alcool ou de drogues étaient également élevés $(3,41$ et 2,68 pour les hommes; 2,35 et 2,07 pour les femmes). Chez les moins de 75 ans, les RT pour les décès qu'une intervention médicale aurait peut-être permis d'éviter étaient de 1,45 pour les hommes et de 1,11 pour les femmes.

Le tableau 5 comprend les TMNA pour les décès toutes causes confondues et pour certains groupes de causes de décès, selon le niveau de compétence professionnel, le groupe d'âge au début de l'étude et le sexe. En ce qui concerne les RT, le gradient de mortalité par niveau de compétence professionnelle était légèrement plus prononcé pour le groupe des 25 à 44 ans (au début de l'étude) que pour celui des 45 à 64 ans. Chez les hommes, le RT était de 2,19 pour les 25 à 44 ans et de 1,72 pour les 45 à 64 ans. Chez les femmes, le RT était de 1,65 pour les 25 à 44 ans et de 1,49 pour les 45 à 64 ans. Alors que les RT d'un niveau de compétence professionnelle à l'autre étaient plus élevés pour le groupe de 25 à 44 ans, c'est chez les 45 à 64 ans que les écarts absolus les plus importants ont été observés.

Les causes de décès ayant contribué le plus à la surmortalité différaient quelque peu selon le sexe et le groupe d'âge. Parmi les membres de la cohorte âgés de 25 à 44 ans, les facteurs ayant contribué le plus à la surmortalité étaient les blessures accidentelles chez les hommes et les cancers de la trachée, des bronches et du poumon chez les femmes. Tant chez les hommes que chez les femmes de 45 à 64 ans, les cancers de la trachée, des bronches et du poumon étaient les principaux déterminants de la surmortalité.

\section{Analyse}

Les gradients de mortalité par niveau de compétence professionnelle étaient évidents pour la plupart des causes de 


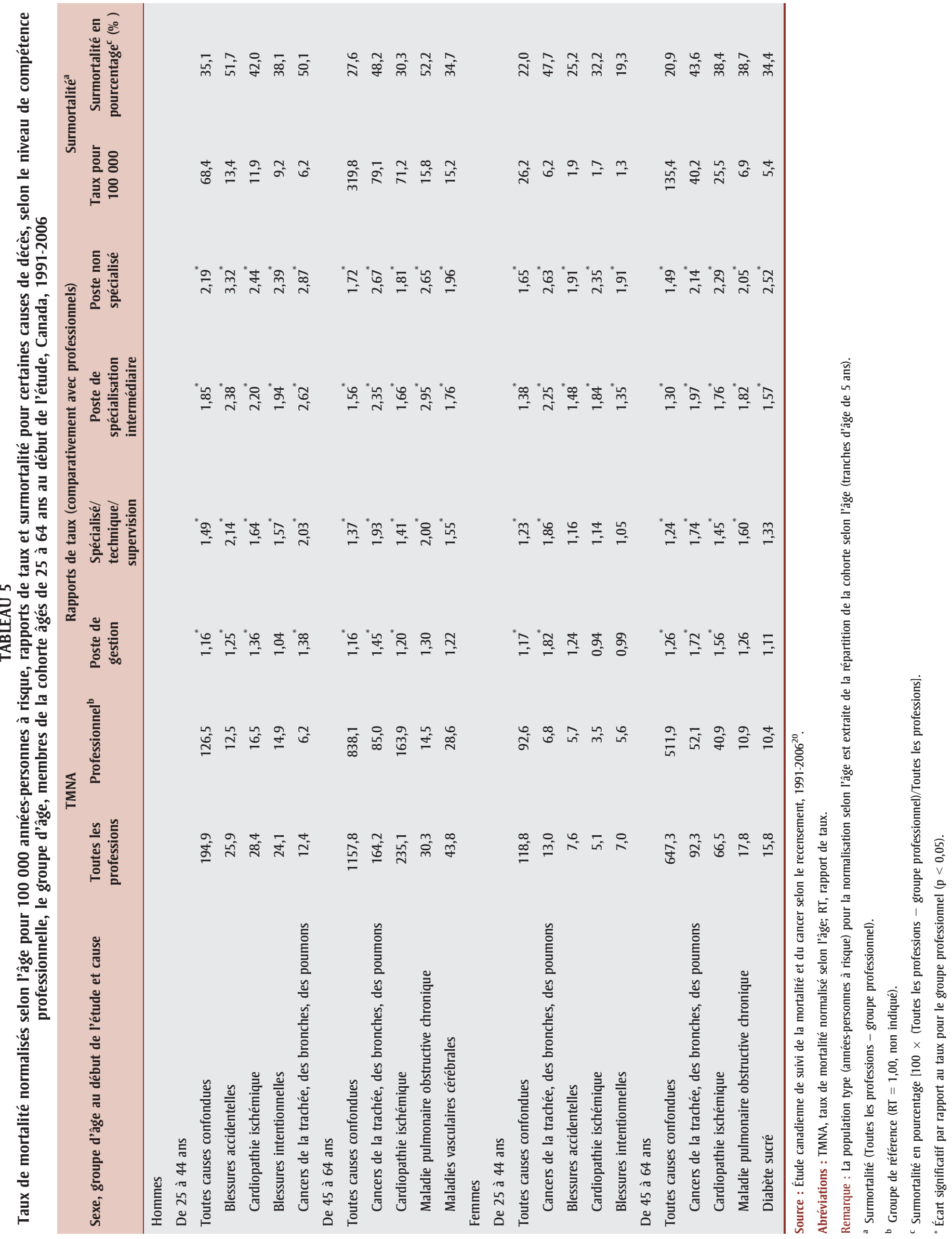


décès, tant chez les hommes que chez les femmes. Nous avons vu que si tous les membres de la cohorte actifs sur le plan professionnel avaient affiché les taux de mortalité normalisés selon l'âge de ceux qui occupaient un poste professionnel, le TMNA toutes causes confondues aurait été de $29 \%$ plus faible chez les hommes et de $21 \%$ plus faible chez les femmes. Chez les hommes, cela correspond à l'élimination de tous les décès par maladie cardiovasculaire et, chez les femmes, à l'élimination de tous les décès par maladie cardiovasculaire et maladie respiratoire.

À quelques exceptions près, il existait un lien entre les taux de mortalité des causes de décès examinées et le niveau de compétence professionnelle. Cependant, le gradient et la force ou magnitude de ce lien variait considérablement en fonction de la cause de décès. Les RT étaient plus élevés pour les causes de décès plus étroitement liées à des comportements à risque pour la santé (comme le tabagisme et la consommation excessive d'alcool), et moins élevés pour les causes de décès qui n'étaient pas étroitement liés à ces comportements (comme les cancers du sein et de la prostate), ainsi que les causes où l'on en sait moins à propos de la prévention. Des études suédoise $e^{6}$ et étatsunienne ${ }^{33}$ ont donné des résultats similaires : Phelan et collab. ${ }^{33}$ ont établi l'existence d'une relation moins forte entre le statut socioéconomique et les causes de décès dans les cas de faible possibilité de prévention. Bien que les liens entre profession et santé soient complexes, et qu'ils aient une incidence tant sur le plan individuel que bionomique $^{34,35}$, les causes de décès les plus évitables avaient tendance à être plus étroitement liées au statut socioéconomique. Sur le plan individuel, cela pourrait être en partie dû au fait que les personnes ayant accès à davantage de ressources seraient en mesure d'adapter leur comportement de façon à tirer profit de nouvelles connaissances au sujet des facteurs de risque et des mesures de prévention $^{36}$.

La réduction des inégalités socioéconomiques en matière de santé est un objectif explicite des politiques canadiennes en matière de santé ${ }^{37}$. L'une des forces de cette étude est que ses résultats sont fondés sur un échantillon large et très représentatif des Canadiens âgés de 25 à 64 ans au moment du recensement de 1991. L'importance de l'échantillon nous a permis d'analyser les différences de mortalité par niveau de compétence professionnelle au sein de groupes détaillés de causes de décès, ainsi que de détecter les effets modestes. Toutefois, la profession connue de chacune des personnes était celle qu'elles exerçaient au début de l'étude (1991) et elle pourrait avoir changé au cours de la période de suivi (1991-2006); elle ne représente donc pas nécessairement le niveau de compétence professionnelle à long terme de ces personnes.

L'étude ne cherchait pas à évaluer l'importance relative des effets directs et indirects de la profession sur la mortalité (par exemple déterminer la part des différences de mortalité par niveau de compétence professionnelle expliquée par des différences associées de revenus et d'éducation). Les données ne contenaient pas d'information sur les facteurs de risque (comme le tabagisme), de sorte que l'effet direct de la profession sur la mortalité a peut-être été surestimé. Néanmoins, d'autres études ont établi que les différences socioéconomiques observées pour divers résultats en matière de santé (y compris la mortalité) persistent en grande partie, même après la prise en compte de l'effet des facteurs de risque comportementaux $^{38-40}$.

\section{Conclusion}

Pour la première fois au Canada, les taux de mortalité par cause ont été examinés de manière approfondie selon le niveau de compétence professionnelle et en fonction d'un large éventail de causes de décès. Les résultats de cette étude confirment ce qui avait déjà été souligné dans des publications internationales au sujet de gradients de la mortalité par niveaux de compétence professionnelle, et ils aident à quantifier l'importance de telles inégalités au Canada.

Nous avons relevé d'importantes différences dans les taux de mortalité selon le niveau de compétence professionnelle pour la plupart des causes de décès. Les causes de décès les plus évitables, dont celles plus étroitement liées au tabagisme et à la consommation excessive d'alcool, affichaient des gradients plus prononcés comparativement aux causes moins évitables. L'Étude de suivi de la mortalité selon le recensement de 1991 à 2006 ayant été élargie afin de permettre l'appariement aux données sur le cancer, de futures études pourraient explorer la nature et l'ampleur des différences en ce qui a trait à l'incidence du cancer et à la survie au cancer selon le niveau de compétence professionnelle.

\section{Remerciements}

Nous tenons à remercier les registraires de l'état civil des provinces et des territoires du Canada pour les données sur la mortalité, Statistique Canada, qui a mené le recensement de 1991, ainsi que tous les Canadiens, dont les réponses fournies au questionnaire complet du recensement ont constitué le fondement de nos analyses. Aucun conflit d'intérêts n'est à déclarer. Le financement de cette étude a été assuré par l'Agence de la santé publique du Canada. L'Étude de suivi de la mortalité selon le recensement a été financée par l'Initiative sur la santé de la population canadienne de l'Institut canadien d'information sur la santé (étude originale), la Direction générale de la santé environnementale et de la sécurité des consommateurs de Santé Canada (prolongations de l'étude) et la Division de l'analyse de la santé de Statistique Canada.

\section{Références}

1. Marmott MG, Bosma H, Hemingway H, Brunner E, Stansfeld S. Contribution of job control and other risk factors to social variations in coronary heart disease incidence. Lancet. 1997;350(9073):235-9.

2. Mackenbach JP, Stirbu I, Roskam AJ et collab. Socioeconomic inequalities in health in 22 European countries. N Engl J Med. 2008;358(23):2468-81.

3. Langford A, Johnson B. Trends in social inequalities in male mortality, 2001-08. Intercensal estimates for England and Wales. Health Stat Q. 2010;(47):5-32. 
4. Saurel-Cubizolles MJ, Chastang JF, Menvielle G, Leclerc A, Luce D; EDISC group. Social inequalities in mortality by cause among men and women in France. $\mathrm{J}$ Epidemiol Community Health. 2009;63(3): 197-202.

5. Weires $M$, Bermejo JL, Sundquist $K$, Sundquist J, Hemminki K. Socio-economic status and overall and cause-specific mortality in Sweden. BMC Public Health. 2008;8:340.

6. Erikson R, Torssander J. Social class and cause of death. Eur J Public Health. 2008;18(5):473-8.

7. Organisation mondiale de la santé, Commission des déterminants sociaux de la santé. Combler le fossé en une génération : instaurer l'équité en santé en agissant sur les déterminants sociaux de la santé : rapport final de la Commission des Déterminants sociaux de la Santé. Genève $(\mathrm{CH})$, Organisation mondiale de la Santé; 2008 [consultation le 15 août 2012].PDF (3,6 Mo) téléchargeable à partir du lien : http://whqlibdoc.who.int/publications/2009 /9789242563702_fre.pdf

8. Stringhini S, Dugravot A, Shipley $M$ et collab. Health behaviours, socioeconomic status, and mortality: further analyses of the British Whitehall II and the French GAZEL prospective cohorts. PLoS Med. 2011;8(2):e1000419.

9. Galobardes B, Shaw M, Lawlor DA, Lynch JW, Davey Smith G. Indicators of socioeconomic position (part 1). J Epidemiol Community Health. 2006;60(1):7-12.

10. Galobardes B, Shaw M, Lawlor DA, Lynch JW, Davey Smith G. Indicators of socioeconomic position (part 2). J Epidemiol Community Health. 2006;60(2):95-101.

11. Lynch J, Kaplan G. Socioeconomic position. Dans : Berkman LF, Kawachi I (dir.). Social Epidemiology. New York: Oxford University Press; 2000. p. 13-35.

12. Mikkonen J, Raphael D. Déterminants sociaux de la santé : les réalités canadiennes. Toronto, York University School of Health Policy and Management; 2010 [consultation le 15 août 2012]. PDF (8 Mo) téléchargeable à partir du lien : http:// www.thecanadianfacts.org/Les_realites _canadiennes.pdf
13. Karasek R, Baker D, Marxer F, Ahlbom A, Theorell T. Job decision latitude, job demands, and cardiovascular disease: a prospective study of Swedish men. Am J Public Health. 1981;71(7):694-705.

14. Shields M. Le stress et la dépression au sein de la population occupée. Rapports sur la santé. 2006;17(4):11-31.

15. Aronson KJ, Howe GR, Carpenter M, Fair ME. Surveillance of potential associations between occupations and causes of death in Canada, 1965-91. Occup Environ Med. 1999;56(4):265-9.

16. Chen J, Beavon D, Wilkins R. Mortality of retired public servants in Canada. Proceedings of the Social Statistics Section, Annual Meeting of the American Statistical Association. Chicago (IL): American Statistical Association; 1996. p. 86-91.

17. Wigle DT, Semenciw RM, Wilkins K, et collab. Mortality study of Canadian male farm operators: non-Hodgkin's lymphoma mortality and agricultural practices in Saskatchewan. J Natl Cancer Inst. 1990;82(7):575-82.

18. Mustard CA, Derksen S, Berthelot JM, Wolfson M, Roos LL. Age-specific education and income gradients in morbidity and mortality in a Canadian province. Soc Sci Med. 1997;45(3):383-97.

19. Wilkins R, Tjepkema M, Mustard C, Choinière R. Étude canadienne de suivi de la mortalité selon le recensement, 1991 à 2001. Rapports sur la santé. 2008;19(3):2648.

20. Peters PA, Tjepkema M. Étude canadienne de suivi de la mortalité et du cancer selon le recensement, 1991 à 2011. Recueil du Symposium 2010 de Statistique Canada. Statistiques sociales: interaction entre recensements, enquêtes et données administratives. Ottawa (Ont.) : Statistique Canada; 2011. p. 150-156. [Statistique Canada, n॰11-522-XCB au catalogue].

21. Mustard CA, Bielecky A, Etches J et collab. Suicide mortality by occupation in Canada, 1991-2001. Can J Psychiatry. 2010;55(6): $369-76$.
22. Mustard CA, Bielecky A, Etches J et collab. Avoidable mortality for causes amenable to medical care, by occupation in Canada, 1991-2001. Can J Public Health. 2010;101(6):500-6.

23. Burrows S, Auger N, Gamache P, Hamel D. Individual and area socioeconomic inequalities in cause-specific unintentional injury mortality: 11-year follow-up study of 2.7 million Canadians. Accid Anal Prev. 2012;45:99-106.

24. Fair M. Generalized Record Linkage System - Statistics Canada's record linkage software. Austrian J Stat. 2004;33(1\&2):37-53.

25. Statistique Canada. Dictionnaire du recensement, 1991. Ottawa (Ont.), Statistique Canada; 1992. [Statistique Canada, n $92-$ $301 \mathrm{~F}$ au catalogue]

26. Emploi et Immigration Canada. Classification nationale des professions : descriptions des professions. Ottawa (Ont.), Groupe Communications Canada; 1993.

27. Spiegelman M. Introduction to Demography. Revised edition. Cambridge (MA): Harvard University Press; 1968.

28. Organisation mondiale de la santé. Manuel de la classification statistique internationale des maladies, traumatismes et causes de décès. $9^{e}$ rév. Genève $(\mathrm{CH})$ : Organisation mondiale de la santé; 1977.

29. Organisation mondiale de la santé. Classification statistique internationale des maladies et des problèmes de santé connexes, $10^{\mathrm{e}}$ rév. Genève $(\mathrm{CH})$ : Organisation mondiale de la santé; 1992.

30. World Health Organization. The global burden of disease: 2004 update. Geneva (CH): World Health Organization; 2008.

31. Deaths related to drug poisoning: England and Wales, 1999-2003. Health Stat Q. 2005 Spring;(25):52-9.

32. Stirbu I, Kunst AE, Bopp $M$ et collab. Educational inequalities in avoidable mortality in Europe. J Epidemiol Community Health. 2010;64(10):913-20. 
33. Phelan JC, Link BG, Diez-Roux A, Kawachi I, Levin B. "Fundamental causes" of social inequalities in mortality: a test of the theory. J Health Soc Behav. 2004;45(3): 265-85.

34. Krieger N. Workers are people too: societal aspects of occupational health disparities an ecosocial perspective. Am J Ind Med. 2010;53(2):104-15.

35. Lipscomb HJ, Loomis D, McDonald MA, Argue RA, Wing S. A conceptual model of work and health disparities in the United States. Int J Health Serv. 2006(1);36:25-50.

36. Phelan JC, Link BG, Tehranifar P. Social conditions as fundamental causes of health inequalities: theory, evidence, and policy implications. J Health Soc Behav. 2010;51(Suppl):S28-40.

37. Agence de la Santé publique du Canada. Rapport de l'administrateur en chef de la santé publique sur l'état de la santé publique au Canada 2008 : S'attaquer aux inégalités en santé. Ottawa (Ont.), Agence de la Santé publique du Canada, 2008. [Santé Canada, n HP2-10/2008F au catalogue].

38. McGrail KM, van Doorslaer E, Ross NA, Sanmartin C. Income-related health inequalities in Canada and the United States: a decomposition analysis. Am J Public Health. 2009;99(10):1856-63.

39. Kim HJ, Ruger JP. Socioeconomic disparities in behavioral risk factors and health outcomes by gender in the Republic of Korea. BMC Public Health. 2010;10:195.

40. Lantz PM, House JS, Lepkowski JM, Williams DR, Mero RP, Chen J. Socioeconomic factors, health behaviors, and mortality: results from a nationally representative prospective study of US adults. JAMA. 1998;279(21):1703-8. 